\title{
A RELEVÂNCIA DE FREIRE PARA ENTENDER O COLONIALISMO ${ }^{1}$
}

RESUMO: O presente artigo é um tributo a Paulo Freire (1921-1997). Mais do que oferecer uma compressão sobre suas ideias que se estendem por quase trinta anos de trabalho, este artigo foca em um aspecto do corpo do seu trabalho. Esse aspecto se insere de perto com o escopo desta publicação: o colonialismo. A ênfase está na "consciência opressora" e na invasão cultural enquanto discussões importantes para o colonialismo, em termos de colonialismo direto e neocolonialismo. Este artigo também desenvolve o complexo tema da linguagem em contextos pós-coloniais.

Palavras-chave: colonialismo; neocolonialismo; consciência opressora; invasão cultural; linguagem; Crioulo.

\section{FREIRE'S RELEVANCE FOR UNDERSTANDING COLONIALISM ${ }^{3}$}

ABSTRACT: This article pays tribute to Paulo Freire (1921-1997). Rather than provide a comprehensive discussion around his ideas from writings spanning around almost thirty years, it focuses on one aspect of his body of work. It is that aspect that closely falls within the purview of this journal: colonialism. The emphasis is on the 'oppressor consciousness' and cultural invasion as they are relevant discussions around colonialism, both in terms of direct colonialism and neo-colonialism. The paper also builds on this theme to address the complex issue of language in postcolonial contexts.

Key words: colonialism; neo-colonialism; oppressor consciousness; cultural invasion; language; creole.

INTRODUÇÃO

O dia dois de Maio de 2020 marca o vigésimo terceiro aniversário da morte de Paulo Freire, um dos maiores referenciais políticos e pedagógicos do século passado cuja influência ecoa fortemente em vários campos. O impacto de Freire na pedagogia é muito conhecido,

\footnotetext{
${ }^{1}$ Tradução de Livia Fernanda Morales e a revisão técnica é da Cleusa Gomes da Silva. Ambas professoras da Universidade Federal da Integração Latino Americana - Unila.

2 Professor da Universidade de Malta. Tem seu trabalho sobre Paulo Freire reconhecido mundialmente, com artigos, livros em traduzidos em diversos países. No Brasil, está traduzido seu livro: Gramsci, Freire e a Educação de Adultos, pela editora Artmed, atualmente esgotado. Endereço Eletrônico: peter.mayo@um.edu.mt

${ }^{3}$ Este artigo foi publicado em Inglês como uma peça In Memoriam, 23 anos depois da morte de Paulo Freire IN: Postcolonial Directions in Education, Vol. 6, No 2, 183-192.
} 
mas sua influência é forte também em áreas como a saúde do trabalho, desenvolvimento comunitário, estudos culturais, comunicação, teologia (Elias, 1994; Leopando, 2017), filosofia e sociologia. Recentemente seu trabalho foi adaptado com resguardos para análise de clássicos literários (Roberts, 2010). A influência de Freire nos estudos pós-coloniais é evidente nos artigos desta publicação. Bastante tem se escrito e continua a se escrever sobre os conceitos básicos da filosofia pedagógica de Freire, portanto evitarei reiterá-los. Esta publicação também tem um artigo que contempla a análise da "Educação bancária" observada pelo filtro da política contemporânea e da política do fazer educacional. No que diz respeito a esta publicação, meu tributo focaliza num aspecto específico dos escritos de Freire - o colonialismo.

Este artigo se baseia na premissa de que o colonialismo ou neocolonialismo toma muitas formas e assuntos que abarcam um "conjunto heterogêneo" de "posições subjetivas" subalternas (Slemon, 1995, p. 45). Já desenhado no meu próprio trabalho anterior (Mayo, 1995, 2004), neste pequeno tributo, vou me restringir ao seguinte: (a) aos conceitos de "consciência opressora" e "invasão cultural" descritos por Carlos Alberto Torres (2014) no livro "Early Freire" e (b) ao complexo assunto da a linguagem na pós independência e na situação pós colonial.

Estas questões aparecem com destaque no proeminente corpo de trabalho de Freire e são discutidas em contextos em que houve colonialismo diretamente, em termos de Edward Said (1993, p.8), o neocolonialismo, e, adotando a perspectiva gramsciana, o "colonialismo interno" que marcam sua presença frequentemente em formas cruéis e violentas. Freire também tem sido relevante em análises sobre educação em situações específicas de "colonialismo colonizador" (Silwadi ad Mayo, 2014; Sperlinger, 2015). Elizabeth Langer (1996) discute as noções de dependência (também analisadas por Torres, 2014) e o papel das igrejas modernizadas de tradição colonial / neocolonial em oposição à "igreja profética" neste contexto. O texto chave aqui é o capítulo destas igrejas em Políticas de educação de Freire traduzido e fortemente discutido por Donaldo Macedo (Freire, 1995). O contraste está no que Cornell West chama de "Igreja Constantiniana" (a "Igreja do Império") e a "Igreja Profética" de orientação popular com base na Teologia da Libertação. Esta é uma teologia definitivamente anticolonial nascida dos contextos mais explicitamente colonizados que deixaram de sê-lo para ser colonizados informalmente pelas superpotências 
como os Estados Unidos e as multinacionais. Dada à natureza colonial e neocolonial do do histórico do trabalho de Freire ${ }^{4}$, não é surpresa que suas ideias sejam frequentemente apresentadas numa posição política pós colonial. Ele representa a política pedagógica para "descolonizar a mente", como primeiro passo no qual o verstehen (entendimento) significa compreender a natureza da opressão e a maneira como a ideologia opera para fazer do ser humano cúmplice da sua própria opressão e a opressão de outros. A imagem do opressor é internalizada pelo oprimido (Freire, 1970, p.30) o que evita a resolução do diálogo dialético oprimido-opressor. Esta situação ecoa na dialética senhor-escravo de Hegel, reinventada dramaticamente por Samuel Beckett através dos personagens Pozzo e Lucky $\underline{5}$ na peça existencialista "Esperando a Godot" (1956).

\section{DIVIDE ET IMPERA - DIVIDIR E GOVERNAR}

Aqueles que são oprimidos em um contexto podem ser opressores em outros. No contexto colonial isto se manifesta nos termos do "dividir e governar", um tema abordado num capítulo inicial da primeira versão da "Pedagogia do Oprimido"6.

A segmentação de linhas raciais/étnicas se manifesta como uma chave contemporânea da estratégia do dividir e governar e implicada no processo de internalização da imagem do opressor. Isto toma importância num período de hegemonia da globalização na que produtores são segregados com bases étnicas e nacionalistas. Também se conecta à ideia de "opressor interno" evidente, por exemplo, na perpetuação de atos de

\footnotetext{
${ }^{4}$ A diferença do controle colonial que se exerce em diferentes países é do mesmo grau em muitos dos países do "Terceiro Mundo", do que Freire trata, como sendo um dos mais atingidos em termos do legado colonial com interseção de classe e raça/etnia.

${ }^{5}$ Ver Raymond Williams (1968, p. 346) nas referências.

${ }^{6} \mathrm{~A}$ intenção original de Freire era a de desenvolver um livro em três capítulos que funcionassem juntos através de um fio condutor dialético. O plano mudou como resultado na insistência do editor de que Freire deveria escrever e incluir um quarto capítulo na luz dos trabalhos de Franz Fanon e Albert Memmi's sobre o colonialismo, assim como o trabalho de outros pensadores (Ver Schugurensky 2011 nas referências). Sempre considerei que a tendência dialética termina no terceiro capítulo e o quarto parece uma inclusão na que temas como 'divide et impera', 'invasão cultural' por si e "unidade para a libertação" são postos como adendos. No entanto, alguns dos temas adequam-se ao contexto colonial da discussão podem, com algum esforço imaginativo, se conectar com os temas profundos tratados nos capítulos anteriores. Relacionar os conceitos de "dividir e governar" ao de "opressor interno" é um exemplo dessa conexão possível entre diferentes temas do livro.
} 
violência contra pessoas cujas características não se enquadram no marco de referência eurocêntricos.

A mentalidade do conquistador.

Esta situação é observável em países ocidentais que desenvolvem sua economia através do trabalho de imigrantes e ao mesmo tempo sendo lugares nos que o medo e a ideia de "concorrência" contra Outros prevalece. Se aplica ao Brasil de Paulo Freire que com uma complexa configuração de política racial engloba brancos, que se posicionam como sendo de origem europeia, até certo ponto pessoas do sudeste asiático e negros e indígenas. Estes últimos são ainda as grandes vítimas da especulação capitalista voraz em áreas como a da Amazônia. Eles são vítimas de um conjunto de atrocidades contemporâneas que Eduardo Galeano viu como continuação da velha mentalidade da "Conquista" (Galeano, 2009). relato arrepiante de Freire sobre o assassinato arbitrário de Galindo Jesus dos Santos, índio Pataxó, incluído na coleção póstumas de ensaios intitulada "Pedagogia da Indignação" (2000), demonstra a continuidade dos atos bárbaros de racismo no Brasil. Este crime em particular é um exemplo da consciência opressora residindo em pessoas que usam a supremacia branca como meio de se posicionar contra a alteridade. Isso lhes dá a sensação de "superioridade posicional", para usar o termo de Said (1978), que permitiria que alguns deles se dessem o direito de matar outros seres humanos por esporte, como se fossem "moscas em mãos de meninos caprichosos" (tradução livre) no famoso verso de Shakespeare em Rei Lear.

Atos violento, racista, sexista, inter-tribais, antropocêntricos ${ }^{7}$ e homofóbicos são exemplos deste tipo de comportamento que indica a presença da "imagem do opressor" no oprimido. Outra vez, este comportamento pode ser incentivado pela estratégia colonial do "dividir e governar" (Freire, 1970, p. 137). É possível apontar como exemplo relativamente recente, dentro de uma variedade de exemplos similares através da história: a carnificina inter-tribal que aconteceu nos anos 90 em Ruanda na que os Tutsis e os Hutus foram colocados uns contra os outros pelo colonialismo Belga.

\footnotetext{
7 Isto inclui a matança desenfreada de animais, insetos (como no verso de Shakespeare) e pássaros - uma contradição dos primeiros trabalhos de Freire (1993) com suas imagens machistas de caça - a conquista da natureza.
} 
A situação é exacerbada pelo processo ao que Freire chamou de "Invasão cultural" a colonização do "universo mental" dos colonizados, nas palavras de Ngũgĩ wa Thiong's (1981, p 16). Isto é educação bancária em larga escala. Foi historicamente caracterizada pela imposição do "arbitrário cultural" (interesses e escolhas culturais) dos colonizadores na maioria dos lugares em que o "conhecimento oficial" (Apple, 1993) foi imposto, especialmente nas escolas. O processo de "anglicização" nas colônias britânicas é um exemplo óbvio. Hoje em dia, a invasão cultural se manifesta através de um tipo perverso do que chamamos de Eurocentrismo Ocidental neocolonial, ecoando a "hegemonia globalizadora" de Boaventura de Sousa Santos, em reconhecimento da presença de um tipo alternativo de globalização: "A globalização de-cima-para-baixo" ${ }^{8}$. Ao mesmo tempo, isto também se apresenta no consumo ideológico da cultura. A "Invasão cultural” foi o processo pelo qual os africanos começaram a ver a si mesmos como "Europeus negros", ou nos termos de Franz Fanon "peles negras em máscaras brancas". Isto faz referência àquele processo que provocou as pessoas da minha nativa Malta a desconsiderar o importante elemento árabe, senão o elemento Islâmico, que participou da história do país e dá ênfase na suposta "linhagem cristã" e "vocação europeia" ininterrupta. Tudo isto pode ser lido como um processo no que certamente sujeitos já diretamente colonizados desejam ser identificados e assimilados no centro do poder europeu colonial.

Claro que discussões em cada um desses contextos se tornam mais complexas quando se reconhece a hibridação como uma traço da vida pós colonial, incluindo as resistências, a assimilação e a apropriação. O termo francês "mestiçagem" é intrigante nesse sentido (ver Tarozzi e Torres, 2016, p. 48)

\section{MEDO À LIBERDADE}

Ecoando Fromm, Freire sustenta que, sob essas condições de prescrição e invasão/dependência cultural, a liberdade é temida pelo oprimido. As pessoas podem se tornar tão domesticadas ao ponto de que nenhuma atividade criativa seja uma possibilidade para elas no caminho do desconhecido. Como argumenta Freire, a criatividade implica no

\footnotetext{
${ }^{8}$ Deve-se ter cuidado com os binários aqui pois os dois se intercruzam. Por exemplo: usar as ferramentas da globalização hegemônica como a internet para fazer com que diferentes ativistas interajam na construção de eventos como protestos de rua ou fóruns sociais mundiais.
} 
risco (Freire e Macedo, 1097, p. 57), com a conotação imediatamente diferente ao conceito de sociedade do "risco" contemporâneo de Ulrich Beck. Arriscar-se é algo de relutância para muitas pessoas que já foram oprimidas pois foram submergidas na "cultura do silêncio". "O país pode sobreviver como uma nação independente?" tem sido uma pergunta frequente com a que me deparei durante a preparação da retirada das forças armadas britânicas de Malta em 1979. Frequentemente tenho ouvido a mesma pergunta de escoceses a luz da possibilidade do Brexit e de outro referendo sobre se a Escócia deve permanecer com a União (este é um país que possuí muitos mais recursos naturais que Malta, desfrutando este último de uma economia dinâmica e um bom padrão de vida).

O medo à "libertação" e ao desconhecido é parte da Educação bancária, um dos conceitos mais famosos de Freire, referenciado no "derramamento da instrução" de Dewey, no que a prescrição é a ordem do dia e as culturas e os espíritos criativos dos colonizados são desabonados e constrangidos como inferiores àqueles dos colonizadores e suas elites consumidoras. É por esta razão, para dar um exemplo, que o governo revolucionário do movimento "New Jewel" de Grenada insistiu na ideia de "grenadizar os grenadenses". Esta foi uma tentativa não de desenvolver um nacionalismo insular mas de incentivar a valorização e orgulho entre a população antes colonizada diretamente (Hickling-hudson, 1997). Foi pensando com o objetivo de ajudar as pessoas a ganhar a confiança necessária para participar do processo de desenvolvimento e fazê-lo de maneira consciente, perguntando-se: Desenvolvimento para quem? Isto repercute no tipo de reflexão central da pedagogia e filosofia da práxis de Freire. A práxis implica uma ação-reflexão de ações transformativas, sendo este não um processo sequencial mas dialético (Allman, 1999).

\section{A QUESTÃO DA LINGUAGEM}

Este é claramente outro aspecto dos escritos de Freire que são de interesse no engajamento de ativistas/adultos envolvidos na política pós-colonial. Este é assunto de linguagem. Freire argumenta que nem tudo o que diz respeito da experiência colonial é irrelevante para o novo contexto pós-colonial. Este ponto é importante quando se considera que a hibridização é uma característica da experiência colonial de resistência ao poder. A "guerra de posição" de Gramsci significa uma apropriação crítica que ocorre em diferentes 
frentes. Freire se refere ao conhecimento da língua do colonizador como benéfico na posição pós-colonial, como no caso das antigas colônias portuguesas tais como Cabo Verde e Guinea Bissau, São Tomé e Príncipe ou Moçambique. Por exemplo: onde e quando as diferentes línguas são usadas por diferentes tribos, a língua do colonizador tem um papel de "língua franca"

Obviamente, o tema da língua nas colonizações diretas ainda é complexo já que os padrões de linguagem da colonização tornam-se fontes de diferenciação social entre grupos e classes, ainda que, em estados pequenos sirva como uma língua de circulação internacional, sendo, portanto, um ativo econômico. Eu afirmaria, no entanto, que o idioma precisa ser ensinado de maneira diferente do que foi feito sob as condições de colonialismo direto. Nas antigas colônias britânicas, a ênfase não seria colocada na 'Anglicização' ou no Inglês ensinado com uma 'missão civilizatória' e com isso conotando ao nativo/indígena como 'incivilizado', mas o ensino de língua como uma experiência libertária.

A política da educação linguística poderia incluir, no contexto pós-colonial, o ensino/aprendizagem das línguas nacionais populares ou originárias e conhecimentos para os que países como o meu já foram considerados "vulgares". Aprender a língua nativa junto com a língua do colonizador pode fazer parte de uma educação bicultural, como defendido a partir da pedagogia crítica com inspiração freiriana por Antonia Darder em relação à educação das e dos latinos nos Estados Unidos (2011). A linguagem colonizada poderia, na perspectiva freiriana, ser ensinada de maneira problematizadora, na que os papéis históricos e sociopolíticos são encarados, problematização que implica em aprender juntos como a ideologia, incluindo a ideologia colonial, que reside nas línguas - um pranto longínquo da forma colonial e neocolonial de ensinar línguas até hoje (ver as discussões sobre TESOL entre imigrantes). Isto também diz respeito ao papel no qual, no caso do Inglês, a língua colonizada é representada como uma força hegemônica (Macedo, Dendrinos e Gounari, 2003). Uma educação direcionada pela práxis implica esta abordagem. Freire (1978), parafraseia Amilcar Cabral quando diz "a linguagem é a expressão cultural mais imediata, autêntica e concreta" (p. 184). Isto ecoa a afirmação de Marx e Engels na 'Ideologia Alemã' de que a linguagem é a "consciência prática" que é tão antiga como a própria consciência. (Marx e Engels, 1970, p. 51) 


\section{LÍNGUAS NACIONAIS-POPULARES}

Dada a importância, no processo da práxis, das línguas originárias e, especialmente, as línguas nacionais-populares, quando existem se transformam em chave à luz da forte relação que entre a linguagem e a consciência, assim como a linguagem e a visão de mundo, como argumenta Gramsci. Freire insistiu que o "fracasso" do seu trabalho em Guinea Bissau foi o resultado do uso do Português "como único veículo de instrução" durante a campanha (Freire em Freire e Macedo, 1978, p.114). Ecoando Pierre Bourdieu, afirma que esse uso torna a linguagem do colonizador em uma forma de "capital cultural", veículo de reprodução do tipo de estratificação de classe (e incluo: raça) associada com a ordem colonial prévia (Freire em Freire e Macedo, 1978, pp. 110-111). Isto explica sua defesa de uma linguagem bilingual/cultural que inclui ao nativo (Crioulo no caso da Guinea Bissau, mesmo que nem todos os setores da população tenham acesso a esta língua híbrida) e a língua do colonizador. Isto deve ser aprendido num sentido problematizador e para propósitos que não empurrem as pessoas à marginalidade da vida política9 ${ }^{9}$

\section{CONCLUSÃO}

As questões da linguagem nas antigas colônias diretas, como com as questões gerais da pós-colonialidade, permanecem complexas. Junto e esboçando com outros autores, Paulo Freire nos ajuda a delinear o caminho desta complexidade. É por isso que, vinte e três anos depois da sua morte, as ideias de Freire continuam inspirando educadores e inúmeros outros pensadores da cultura.

\section{REFERÊNCIAS}

ALLMAN, P. Revolutionary Social Transformation: Democratic Hopes, Political Possibilities and Critical Education. Westport CT: Bergin \& Garvey, 1999.

\footnotetext{
${ }^{9}$ Esta situação lembra a discussão de Gramsci sobre a linguagem padrão e dialetos/ letramentos na busca do desenvolvimento de uma língua nacional-popular.
} 
APPLE, M. W. Official Knowledge: Democratic Education in a Conservative Age. New York and London: Routledge, 1993.

BECKETT, S. Waiting for Godot. London: Faber and Faber, 1956.

DARDER, A. Culture and power in the classroom: a critical foundation for bicultural education. 2 Ed. Previously Boulder Co: Paradigm; now New York and London: Routledge, 2011.

ELIAS, J. Paulo Freire: Pedagogue of Liberation. Malabar, FL: Krieger, 1994.

FREIRE, P. Pedagogy of the Oppressed. New York: Continuum, 1970.

FREIRE, P. Education for Critical Consciousness. New York: Continuum, 1973.

FREIRE, P. Pedagogy in Process. The Letters to Guinea Bissau, New York: Continuum, 1978.

Freire, P. Politics of Education. Culture, Power and Liberation. (D. Macedo trans.), Massachusetts: Bergin \& Garvey, 1985.

FREIRE, P. Pedagogia da indignação: cartas pedagógicas e outros escritos. São Paulo: Editora UNESP, 2000.

FREIRE, P. and Macedo, D. Literacy. Reading the Word and the World. Westport CT: Bergin \& Garvey, 1987.

Galeano, E. Open Veins of Latin America. Five Centuries of the pillage of a Continent, London: Serpent's Tail, 2009.

HICKLING-HUDSON, A. A Caribbean Experiment in Education For Social Justice. In T. Scrase, Social Justice and Third World Education, pp. 133-162, New York and London: Garland Publishing, 1997.

LANGE, E. Freire and Liberation Theology. In H. Reno and M. Witte (eds.) 17th Annual Adult Education Research Conference, Proceedings 1996, Tampa FL: University of South Florida, 1996.

LEOPANDO, I. A Pedagogy of Faith. The Theological Vision of Paulo Freire, New York and London: Bloomsbury Academic, 2017.

MACEDO, D. DENDRINOS, B. and GOUNARI, P. The Hegemony of English. prev. Boulder Co: Paradigm; now New York and London: Routledge, 2003.

MARX, K. and ENGELS, F. The German Ideology. (C.J. Arthur ed.), London: Lawrence and Wishart, 1970. 
MAYO, P. Critical Literacy and Emancipatory Politics. The Work of Paulo Freire. International Journal of Educational Development. 15, 1995, p. 363-379.

MAYO, P. Liberating Praxis. Paulo Freire's Legacy for Radical Education and Politics, Westport CT: Praeger, 2004.

Ngũgĩ wa Thiong'o. Decolonizing the Mind. The Politics of language in African literature Oxford: James Currey \& Heinemann, 1981.

ROBERTS, P. Paulo Freire in the 21st Century. Education, Dialogue and Transformation, New York and London: Routledge, 2010.

SAID, E. Orientalism. New York: Random House, 1978.

SAID, E. Culture and Imperialism. London: Vintage, 1993.

Schugurensky, D. Paulo Freire. New York and London: Continuum, 2011.

SILWADI, N. and MAYO, P. Pedagogy Under Siege in Palestine: Freirean Approaches. Holy Land Studies. Vol. 13 (2): p. 71-87. 2014.

SLEMON, S. The Scramble for Post-colonialism. In B. Aschroft, G. Griffiths and H. Tifflin (eds.) The Postcolonial Studies Reader, London and New York: Routledge, 1995.

SPERLNGER, T. Romeo and Juliet in Palestine. Teaching under Occupation, Winchester: Zero Books, 2015.

TAROZZI, M. and TORRES, C.A. Global Citizenship Education and the Crises of Education Reform. London and New York: Bloomsbury Academic, 2016.

TORRES, C.A. The First Freire. Early Writings in Social Justice Education, New York: Teachers College Press, 2014.

WILLIAMS, R. Drama from Ibsen to Brecht. Harmondsworth: Pelican, 1968.

Recebido em 18 de abril de 2020.

Aprovado em 26 de outubro de 2020.

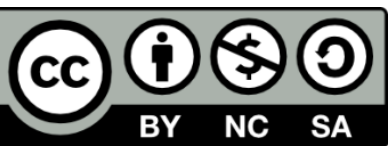

